\title{
La inmaterialidad y normatividad del patrimonio cultural
}

\section{The immateriality and normativity of intangible cultural heritage}

Mercedes Mariano mercedes.mariano@gmail.com

http://orcid.org/0000-0002-7052-9597 Instituto de Investigaciones Arqueológicas y Paleontológicas del Cuaternario Pampeano; Universidad Nacional del Centro de la Provincia de Buenos Aires y Consejo Nacional de Investigaciones Científicas y Técnicas (Argentina)

\section{Resumen}

El presente artículo tiene como objetivo analizar la producción social del patrimonio cultural inmaterial como un campo que es atravesado por lógicas normativas. En este sentido, se busca abordar el patrimonio cultural inmaterial como un campo (en los términos de Bourdieu) y analizarlo a la luz de los aportes de la antropología para así poder llevar a cabo una lectura que vuelva visible la heterogeneidad omnipresente de prácticas, ideas jurídicas, definiciones, conductas y normas. Para ello se recuperarán algunas de las normativas vigentes internacionales y nacionales- para comprender el conjunto de las disputas que refuerzan o limitan en cada contexto la vigencia del mismo, así como sus múltiples interpretaciones.

Palabras clave: patrimonio inmaterial; normatividad; campo; antropología. 
Abstract

The objective of this article is to analyze the social production of intangible cultural heritage as a field that is crossed by normative logics. In this sense, it seeks to address the intangible cultural heritage as a field (in the terms of Bourdieu) and analyze it in light of the contributions of anthropology in order to carry out a reading that makes visible the omnipresent heterogeneity of practices, ideas legal, definitions, behaviors and standards. To this end, some of the current regulations -international and national- will be recovered to understand the set of disputes that reinforce or limit the validity of the same in each context, as well as its multiple interpretations

Keywords: intangible heritage; normativity; field; anthropology.

En 2013 publiqué junto a la Doctora María Luz Endere un artículo que reflexionaba sobre la protección del patrimonio cultural inmaterial a partir de un análisis sobre su conceptualización actual, abordándolo en diferentes escalas que contemplaban el plano internacional, regional y local (Mariano y Endere, 2013). Allí dimos cuenta cómo desde el siglo XIX, los estados nacionales se preocuparon por proteger algunos componentes de su patrimonio cultural material a través de la legislación (e.g. Leyes de Monumentos Nacionales) mientras que la protección del patrimonio cultural intangible se vio postergada hasta mucho tiempo después (siglo XXI).

Contar con instrumentos legales capaces de proteger el patrimonio intangible en su totalidad se volvió una necesidad en un contexto internacional, caracterizado por un vasto reconocimiento de los derechos de las "minorías" étnicas. Algunos ejemplos de ello fueron: el Convenio 169 sobre pueblos indígenas y tribales en países independientes de la Organización Internacional del Trabajo (OIT) de 1989 y la Declaración de las Naciones Unidas sobre los derechos de los pueblos indígenas de 2007; la aceptación de la incidencia de la diversidad cultural en la manera de concebir, conservar y gestionar el patrimonio como se manifestó en la Carta de Burra de ICOMOS Australia y en la Conferencia de Nara sobre autenticidad de UNESCO, 1994, entre otras (Mariano y Endere, 2013: 34-35).

La Convención para la Salvaguarda del Patrimonio Cultural Inmaterial en 2003, en el seno de la UNESCO y la posterior ratificación por parte de los Estados Miembros, generó la necesidad de contemplar la protección de dicho patrimonio en el sistema legal de cada país y de ampliar su alcance conforme a la nueva normativa internacional. En este marco, en 2006 se implementa el 
Centro Regional para la salvaguarda del Patrimonio Cultural Inmaterial de América Latina (CRESPIAL), un organismo internacional creado por iniciativa del gobierno de Perú, con el apoyo de la UNESCO, para promover acciones de salvaguardia y protección del vasto patrimonio inmaterial de los pueblos latinoamericanos.

La República Argentina, mediante la Ley Nro. 26118/06 ratificó la Convención UNESCO de 2003, y la Secretaría de Cultura de la Presidencia de la Nación designó a la Dirección Nacional de Patrimonio y Museos como el órgano encargado de llevar a cabo la salvaguardia de este patrimonio en el país. Esta dirección, a su vez, creó el Programa Nacional de Patrimonio Inmaterial cuyo objetivo se centra en la identificación, inventario y registro del patrimonio, incorporando un diseño y líneas generales de acción basados en la experiencia realizada en Brasil (Mariano y Endere, 2013). La particularidad argentina es que cada provincia tiene la potestad jurídica de sancionar normas, así como de proponer y ejecutar diferentes políticas de gestión para fomentar la salvaguardia y puesta en valor del patrimonio inmaterial, conforme a las facultades otorgadas por el artículo 41 de la Constitución Nacional. Esto permite que coexistan en un mismo territorio diferentes normas de protección, con criterios y alcances diversos en relación al patrimonio intangible. Todas estas convenciones, organismos, normas y leyes, si bien lo hacen de manera diferente, definen qué es el patrimonio cultural inmaterial y promueven acciones para su salvaguarda.

Por su parte, el patrimonio cultural comenzó a ser tema de interés académico. Esto se tradujo en un creciente corpus de conocimientos y estudios centrados en el patrimonio. El tema fue, y es, analizado por una diversidad de disciplinas científica e incluso desde perspectivas interdisciplinares. La incursión de la antropología en la temática posibilitó un nuevo tipo de discusiones. En América Latina el replanteamiento del tema fue impulsado por el antropólogo García Canclini, la antropología brasileña y por los trabajos de Bonfil Batalla (1989). En la actualidad y, como resultado de los debates previos mencionados, resulta inverosímil centrar los análisis de los bienes culturales aislados de su proceso de producción y circulación social. De esto modo, se reconoce al patrimonio en tanto construcción/producción sociocultural y se lo entiende como ámbito de enfrentamientos y negociaciones, así como recurso para reproducir identidades y también, diferencias sociales (Rosas Mantecón, 1998).

En este contexto más amplio, el concepto de patrimonio cultural inmaterial comenzó a englobar una serie de temas que fueron y son estudiados por la antropología y más específicamente desde la perspectiva del folklore (Martin, 2007). Son recientes los estudios que abordan estas prácticas desde la perspectiva del patrimonio cultural intangible en la Argentina. Caben destacarse los trabajos vinculados con las manifestaciones culturales festivas, carnavalescas y las murgas en la ciudad de Buenos Aires (Crespo, 2007; Martin, 1997, 2001; Canale, 2007; 
Morel, 2007, 2011; Canale y Morel, 2005; Rotman, 2001, 2004, 2010); así como aquellos relacionados con expresiones de inmigrantes y sus descendientes (Gavazzo, 2005; Lacarrieu, 2010; Mariano, 2011). Sin embargo, no son tan comunes los trabajos que abordan el patrimonio inmaterial desde la perspectiva de la antropología jurídica, cuando en realidad, la impronta burocrática del mismo hace del entramado de normativas que lo constituyen una dimensión de análisis de primer orden.

Por todo ello, en este artículo me propongo abordar la producción social del patrimonio, sus administraciones y su dimensión performativa, a través de un análisis que permita pensar al patrimonio inmaterial como un campo, en los términos de Bourdieu (2000).

\section{El patrimonio inmaterial en su definición}

El patrimonio cultural inmaterial se convirtió entonces en un tema de interés en la campo de las Ciencias sociales y se lo comenzó a entender como una categoría conceptual, como fuente de diversidad, identidad, creatividad y, además, como práctica y conocimiento de quienes lo portan (Bouchenaki, 2004; Kirshenblatt-Gimblett, 2004; Kurin, 2004; Sánchez-Carretero, 2005; Blake, 2009, 2014; Lacarrieu, 2010; Mariotti, 2011; Higginbottom, 2014). Su conceptualización e institucionalización se enmarca en la Convención para la Salvaguarda del Patrimonio Cultural Inmaterial de la UNESCO adoptada en 2003, en donde se lo definió como:

\footnotetext{
los usos, representaciones, expresiones, conocimientos y técnicas (...) que las comunidades, los grupos, y en algunos casos los individuos reconozcan como parte integrante de su patrimonio cultural" (art. 2.1). El mismo puede manifestarse en "las tradiciones y expresiones orales, en las artes del espectáculo, en los usos sociales, rituales y actos festivos, en los conocimientos y usos relacionados con la naturaleza y el universo, así como en las técnicas artesanales tradicionales (art. 2.2). Se entiende por salvaguarda a todas aquellas medidas destinadas a garantizar la viabilidad del patrimonio cultural inmaterial, comprendiendo las acciones de identificar, documentar, investigar, preservar, proteger, promocionar, valorizar, transmitir -básicamente a través de la enseñanza formal y no formal- y revitalizar el patrimonio en sus distintos aspectos (Artículo 2.3).
}

Ahora bien, interesa en primera instancia analizar las implicancias político culturales que las definiciones (su materialización en un texto escrito ya sea en una norma, ley o convención) establecen ¿Qué tipo de cuestiones resuelve? Como lo expresa Krotz (2002) "Toda definición es un proceso de delimitación que tiene dos caras: identifica las características de un 
fenómeno que es parte de un todo mayor y, al mismo tiempo, señala la ubicación de esta parte en este todo mayor" (p. 25).

En este marco, es posible advertir cómo los textos y las palabras crean presencia (Díaz Cruz, 2012; Austin, 1970) consagran verdades, resuelven ambivalencias, codifican, fijan, oficializan el saber, un saber. Recuperando lo que expresa Díaz Cruz (2012) "hacen, actúan, producen y realizan, son proyectiles verbales" (p. 41). En general son el resultado de una disputa previa por legitimar cierta versión de los hechos que se resuelve y se formaliza en un texto. Una vez escrito, se elimina la controversia anterior, pero se delimita el campo para nuevas disputas. "Hacer cosas con palabras" (Austin, 1970) constituye una posibilidad de la performance (Díaz Cruz, 2012).

La performance es un hacer que describe ciertas acciones que están transcurriendo, ejecutadas en sitios específicos, atestiguadas por otros o por los mismos celebrantes: es un hacer que focaliza esa presencia en acto de creación. Pero también nos retrotrae a lo ya hecho, a performances completadas, concluidas, recordadas, olvidadas y vueltas a recobrar, que atraviesan e implican campos discursivos, textos, pre existentes (...) Al mismo tiempo que es un transcurrir, la performance hace inteligible la materia, las habilidades, los tópicos, elementos, textos, objetos y reglas con que se construye, y de los que estuvieron armadas previas performance (Díaz Cruz, 2012: 44).

Esta capacidad de poder nombrar, representar, crear presencia, instituir realidades mediante el lenguaje, es netamente cultural y social. Esto significa, retomando nuevamente a Krotz (2002), que el universo de la especie humana de todos los tiempos y todos los lugares es para la antropología "un multiverso sociocultural" (2002: 36).

Las activaciones patrimoniales (algunas de ellas traducidas en leyes, normas o convenciones), escribe Prats (2006) y el mismo concepto de patrimonio, nacieron para sacralizar y expresar un conjunto de discursos identitarios mantenidos por el poder de turno. "El patrimonio nunca ha sido activado desde el consenso social, sino básicamente desde los intereses políticos" (2006: 74).

El patrimonio entonces es una imagen, es la representación de una identidad, no la identidad misma. El patrimonio activado está formado por el conjunto de representaciones más o menos sesgadas de la realidad, pero no es la realidad (2006: 75) es una versión de ella.

Al margen de las activaciones patrimoniales, las sociedades viven y evolucionan, elaboran y reelaboran, comparten y disientes versiones de la identidad (...) Las activaciones patrimoniales son un elemento más en la autoconstrucción ideológica de la comunidad, no la plasmación de esta (...) La población distingue claramente vida de patrimonio (Prats, 2006: 75). 
No obstante, aunque el patrimonio no sea la identidad, sus referentes mantienen un carácter de sacralidad para el grupo. En suma, como lo expresa Agudo Torrico:

toda imagen creada o por crear sobre lo que ha de formar parte del patrimonio, sus niveles de relevancia, acciones destinadas a su valorización, protección (...) no deja de ser una creación cultural, una construcción social vinculada con períodos históricos precisos y a los discursos ideológicos imperantes en cada momento (2003: 13).

Por lo hasta aquí expuesto es que toda delimitación y definición de lo que es patrimonio cultural (y lo que no), no debe ser tomado con naturalidad ni ingenuidad, sino como el resultado de procesos en los que intervienen actores sociales e instituciones que tienen el poder y el capital para legitimar determinadas ideas, realidades, imágenes y sentidos sobre los patrimonios. La UNESCO, a través de sus declaratorias, normativas y Convenciones, se instituye como "el" organismo con carácter de experto, como "la autoridad legítima que determina y otorga reconocimiento a los referentes nacionales que pretenden integrar sus bienes culturales a las 'Listas' de patrimonio" (Rotman, 2015: 19). La legitimidad construida en torno a esta institución genera una acepción acrítica de sus enunciados y políticas, y al mismo tiempo, genera prestigio simbólico.

A partir de la perspectiva antropológica se busca dar cuenta de la manera en que los sistemas y las instituciones, así como las definiciones que producen, se encuentran inmersas en la cultura y el poder. En este sentido a través de ella se pretende explica los fenómenos que estudia, mediante la búsqueda de aquellas estructuras que subyacen a lo observable. Por todo ello, el fin es problematizar las definiciones de patrimonio cultural inmaterial identificando el sistema de normas que promueve su halo de naturalidad e inmutabilidad.

\section{El patrimonio cultural inmaterial como campo jurídico}

Bourdieu (2000) define un campo como un sistema de disposiciones sociales que se definen unas en relación con otras, o como bien lo sintetizan Moreno y Ramírez:

Un espacio específico en donde suceden una serie de interacciones (...) un sistema particular de relaciones objetivas que pueden ser de alianza o conflicto, de concurrencia o de cooperación, socialmente definidas e instituidas, independientes de la existencia física de los agentes que la ocupan (2003: 16). 
Por su parte define el campo jurídico como un campo de batalla en el que se lucha por el monopolio que da a los agentes la potestad de decidir qué es derecho y qué no. Lo característico de este campo es la ilusión de neutralidad y de autonomía que constituye discursos y rige prácticas, construyendo imaginarios, representaciones y redes de significación de los ocupantes del campo (Bourdieu, 2000; Fortich Navarro y Durán, 2012).

Para que funcione un campo, es necesario que haya algo en juego y agentes dispuestos a jugar; que esté dotado de los habitus que implican el conocimiento de las leyes inmanentes al juego. De hecho, para que un campo pueda determinarse como tal, debe tener agentes denominados "profesionales" (sujetos expertos en esa materia) y unos profanos (que no tienen ni el conocimiento de las habilidades certificadas por los "guardianes" del campo). Todos los campos están mediados por los profanos y por los profesionales que entran y salen (Fortich Navarro y Durán, 2012: 51).

El campo es entonces una estructura donde se da una lucha, de unos agentes que han internalizado las reglas del juego y donde hay unos capitales específicos. Ahora bien ¿cuáles son las reglas del juego en el campo del patrimonio intangible? ¿Qué capitales entran en escena y cómo se vinculan con los "profesionales" y los "profanos"?

El patrimonio cultural es arena de lucha. Los sentidos que a través de él se ponen en juego, no responden a un único orden ni lógica. Igual que en un campo, hay determinados actores que por medio de su capital económico y político intentan imponer un sentido sobre los otros. No implica un consenso unánime sino que es un espacio de contestación (Van Zanten, 2004: 37), ya que, en tanto bien cultural, es "praxis, abierta y atravesada por relaciones de poder, que pueden generar la naturalización de lo arbitrario, así como la puja por hacer emerger o recrear significados alternativos" (Briones, 1998: 6). Por ello, autores como Gravano señalan que "los significados no son algo dado sino construcciones permanentes", es decir que, "ningún signo se mantiene o se trata de mantener de modo inercial, sin una razón histórica, sin un interés o una racionalidad que lo motoriza" (2008: 12). Como para el caso del campo jurídico, en el del patrimonio, el mantenimiento de determinados valores y reglas depende de quién detente el poder de establecer esos sentidos o de convencer a los demás de que estos son los que deben predominar.

En este contexto, se podría establecer cierta analogía con aquellos actores que, dentro de instituciones particulares como la UNESCO, definen qué es, y qué no, patrimonio cultural inmaterial. Ya que, como en los campos que propone Bourdieu, allí hay "profesionales" con el 
capital de intervenir en la elaboración de Convenciones Internacionales y de materializar en un texto un conjunto de normas y reglas que responden a una lógica "experta".

Los sistemas de reglas, como lo expresa Krotz (2002) "incluyen enunciados formalizados pero también implican la generalización, aplicación, interpretación, vigilancia y modificación de tales enunciados, así como de las instituciones, los cargos especializados y los actores sociales involucrados en todo ese proceso" (2002: 33). Esta lógica es traducida a una estructura determinada que se plasman en una Convención (en este caso la de 2003) a través de títulos como "disposiciones generales", artículos y "considerandos", donde a su vez se delimitan las acciones y prácticas para la salvaguarda de dicho patrimonio.

La misma idea de campo supone entonces una "re-semantización del lenguaje, ya que cada uno tiene un metalenguaje que define sus códigos y confiere poder dentro del campo a quienes se apropian de él y lo manejan" (Navarro y Durán, 2012: 52). Dicho lenguaje, en este caso puntual, es un capital para los profesionales dentro del campo del patrimonio.

Sin embargo, reconocer al patrimonio cultural inmaterial en los términos de la Convención de 2003 no fue una tarea sencilla (Kurin, 2004). Mucho se ha discutido y publicado sobre esto, no obstante aquí se busca remitir a aquellos puntos más significativos a la hora de generar consensos en una definición. En primer lugar se debió llegar a un acuerdo respecto del uso del término "intangible" (en inglés) por las dificultades que encontraron "los expertos" en adoptar otros posibles, tales como folklore, patrimonio oral, cultura tradicional, cultura expresiva, modo de vida, cultura etnográfica, costumbres, cultura popular o patrimonio vivo cultural. "A las dificultades epistemológicas (...) se unen las cargas ideológicas de los mismos, las particulares historias disciplinarias en cada país y las complicaciones para traducir conceptos, tanto por lo que denotan como también por lo que connotan" (Sánchez Carretero, 2005: 149).

No obstante, las nociones de "inmaterial" y "material", o de "tangible" e "intangible" (en inglés), no resuelven la cuestión, es más, plantean un binarismo implícito (Ibídem: 151) que no tiene sentido dentro de determinadas lógicas culturales (Sánchez Carretero, 2005; Dawson, 2004; Boychenaki, 2004; van Zanten, 2004; Kurin, 2004).

Por su parte, el mismo concepto de "salvaguardia" fue objeto de cuestionamientos. Salvaguardar implica garantizar la viabilidad del patrimonio cultural inmaterial. No obstante, y entendiendo el patrimonio como producción sociocultural (histórica y cambiante) se comenzó a plantear que ninguna intervención debería 'garantizar' ese resultado. "La cultura cambia y los usos del pasado se desechan cuando dejan de ser funcionalmente útiles o simbólicamente importantes para una comunidad" (Kurin, 2004: 78). Este punto cobra especial relevancia en la misma idea de génesis del campo que propone Bourdieu, ya que implicaría una operación dialéctica. Para dicho autor el campo tiene una dinámica, por lo que el mismo nunca está 
quieto. Sin embargo, es frecuente que quienes tienen el poder ("los expertos"), se preocupen por generar una estática, es decir, una especie de fuerza que lo retenga estable y lo legitime ("garantice la salvaguardia"). Por todo ello pensar el patrimonio como un campo, permite visibilizar la producción de una lucha de fuerzas entre quienes no tienen el poder ("los profanos") que promueven la dinámica y quienes sí lo tienen y procuran su funcionamiento estático (Fortich Navarro y Durán, 2012: 52-53).

En suma, el concepto de campo en Bourdieu se ilustra como un espacio de juego autónomo con reglas que los agentes deben conocer para entrar, permanecer y salir del campo. "Esas reglas las deben conocer e interiorizar los diversos agentes para poder jugar y procurar la existencia y sobrevivencia del mismo. Son reglas que quienes están jugando, convierten en normas" (Fortich Navarro y Durán, 2012: 53). En este punto resulta interesante recuperar el tema de los conocimientos tradicionales, ya que los mismos podrían enmarcarse dentro del campo del patrimonio inmaterial y porque presentan diversas aristas en relación a aspectos conceptuales, epistemológicos, políticos, jurídicos y económicos que se dirimen en el juego.

Los conocimientos son una producción colectiva y creativa que generalmente se transmite por medio de la oralidad y que se caracterizan por ser eminentemente empíricos. A nivel internacional no existe una definición compartida y aceptada de "conocimientos tradicionales". No obstante, representantes de comunidades indígenas han definido a dichos conocimientos como "aquellos que poseen los pueblos indígenas y comunidades locales, transmitidos de generación en generación, habitualmente de manera oral y desarrollados al margen de la educación formal que imparten los Estados" (Coordinadora de las Organizaciones Indígenas de la Cuenca Amazónica, 1999: 9). Estos saberes son intangibles e integrales a todos los conocimientos y prácticas ancestrales, por lo que constituyen el patrimonio intelectual colectivo y se vinculan con las ciencias naturales, los rituales, los cantos, las danzas y ritmos, las artesanías, las curaciones, la medicina y farmacología, entre muchos otros (Endere y Mariano, 2013).

La Organización Mundial de la Propiedad Intelectual (en adelante OMPI) se refiere a los conocimientos tradicionales como aquellos trabajos artísticos, literarios y científicos basados en la tradición, que incluyen actuaciones, invenciones, diseños, marcas, descubrimientos científicos, nombres, símbolos, información no divulgada y cualquier innovación y creación que resulte de una actividad intelectual, basada en esa tradición.

Conforme a los criterios desarrollados por la OMPI, los conocimientos tradicionales para poder ser protegidos mediante mecanismos jurídicos, tendrán que: a. haberse creado y preservarse en un contexto tradicional y transmitirse de una generación a otra; b. estar particularmente 
vinculados a un pueblo o comunidad indígena o tradicional, que los preserva y transmite de una generación a otra; y c. ser parte integrante de la identidad cultural de un pueblo o comunidad indígena o tradicional que es reconocido como su titular porque sobre ellos ejerce su custodia, conservación, titularidad colectiva o responsabilidad cultural (OMPI, 2008).

Numerosos pueblos y comunidades indígenas han alzado sus voces para demandar a los Estados que pongan freno a la biopiratería, es decir "al acceso y uso de recursos biológicos y conocimientos asociados sin el consentimiento de sus poseedores ni una adecuada compensación por su utilización" (Ruiz, 2001: 78). Se trata de un concepto político más que legal e implica una condena de tipo moral por ser un campo escasamente regulado.

Otra importante amenaza es la pérdida del control por parte de las comunidades sobre sus conocimientos tradicionales por haber pasado al campo del dominio público, cuestión en la cual ha sido clave el papel de los propios científicos que han identificado e incluido en sus bases de datos y publicaciones dichos conocimientos (Endere y Mariano, 2013: 7).

La mayoría de las comunidades indígenas "no comparten las nociones individualistas de propiedad privada que tienen la mayoría de los países de Occidente y, por lo tanto, no están de acuerdo con la existencia de límites temporales de protección" (Tobón Franco, 2006: 96). Esto entra en contradicción con la naturaleza misma de un conocimiento que es compartido, así como con el sistema de creencias y prácticas indígenas. Sin embargo, cuando las comunidades ven vulnerados sus derechos y deciden reclamar por sus conocimientos tradicionales, están optando por una concepción política de estos saberes, están apropiándose del sistema de reglas del juego para poder defenderse de, por ejemplo, compañías globales que pretenden comercializarlos.

\section{La legitimación del campo}

La Convención para la Salvaguardia del Patrimonio Cultural Inmaterial de 2003 generó un impacto considerable no sólo en el sistema de la UNESCO, que incorporó la protección de este patrimonio en todos sus programas, sino también en otras recomendaciones de expertos y en las políticas de los diferentes países miembros. La Carta de Ename para la Interpretación de Lugares Pertenecientes al Patrimonio Cultural (ICOMOS, 2005) fue un ejemplo de ello ya que estableció que los elementos inmateriales de un lugar con valor patrimonial deben identificarse e incluirse" en la interpretación de dichos lugares (Principio 3.5). En igual sentido, la Carta de Itinerarios Culturales de ICOMOS (2008) que estableció que "los contenidos intangibles de un Itinerario Cultural son fundamentales para comprender su sentido y sus valores patrimoniales 
de carácter asociativo y que, por lo tanto, los aspectos materiales deben relacionarse siempre con otros valores de naturaleza intangible".

De este modo, se comenzó a visibilizar desde estos organismos (UNESCO, ICOMOS), la importancia que reviste el patrimonio cultural inmaterial como crisol de la diversidad cultural y como garante del desarrollo sostenible. Sin embargo como señala Rotman:

el patrimonio como categoría ha ido atravesando un proceso intenso de constante expansión durante las últimas décadas (...) La continua complejidad adquirida por el campo patrimonial, requiere de un esfuerzo intelectual que posibilite la construcción de perspectivas que apunten a sustentar y viabilizar la capacidad analítica de la categoría, al mismo tiempo que tiendan a mantener, a asegurar su capacidad como estrategia, como recurso de confrontación, de demanda, de sectores sociales que bregan y disputan por su reconocimiento y legitimación, dado que, como se ha explicitado, el patrimonio es permeado por la desigualdad, el conflicto y el poder (2015: 21).

En este sentido, el patrimonio es un proceso de integración que se encuentra permanentemente en tensión y amenazada por fuerzas disruptivas. Uno de los mecanismos esenciales para garantizar y reforzar su permanencia y reproducción, afirma Krots (2002) -pero para referirse a la sociedad-, es la esfera del derecho y, como se vio hasta aquí, tal esfera es la de cierto tipo de reglas. El sistema de reglas se ocupa, ante todo, de conflictos y, en definitiva, refleja las características socio económico y político fundamentales de la realidad de la que forma parte. A través de él se expresan los valores aceptados consensualmente por la sociedad. Como cualquier sistema normativo, no es un fenómeno del orden natural sino creación histórica de los seres humanos (Krtoz, 2002). Por todo ello también tienden a ser conflictivos en su misma dinámica ya que no suelen resolver la totalidad de las tensiones sino legitimar un conjunto de respuestas provisorias para cuestiones que son jerarquizadas para un grupo.

Ahora bien, "todos los campos necesitan legitimarse". En este sentido, el derecho es un visitante en todos los campos de la sociedad (incluso en el patrimonial), porque él regula y legitima. Todo tiene que estar reglado, definido y explicitado ¿qué es legal y que no? ¿Qué es patrimonio y qué no? (Fortich Navarro y Durán, 2012: 53).

Para el caso puntual de campo del patrimonio cultural inmaterial dicha legitimación se produce por medio de rituales particulares que construyen universos de jerarquías y funciones y que configuran un orden. En este sentido, Geertz sostiene que los rituales implican procesos de simbolización donde se recombinan los elementos de la cotidianeidad (prácticas, 
manifestaciones, saberes, entre otros bienes de orden inmaterial) a través de un proceso de deslocalización que, a través del rito, produce en dichos elementos un efecto de focalización (Kaufman, 1991). Mediante esta puesta en foco, expresa Kaufman, se establecen contrastes dentro de mundo cotidiano, destacando nuevas fronteras sociales y anulando otras, subrayando algunas identidades y pasando a un plano secundario a otras. Es decir, una vez que una manifestación cultural (un conocimiento tradicional, una manifestación festiva, entre otras) comienza a ser pensada en los término de patrimonio cultural inmaterial, empieza a ser reelaborado en función de una gramática y una normativa y un conjunto de ritos, que lo colocan en un plano diferente del ordinario para re significarlo y convertirlo en otra cosa.

Siguiendo a Bourdieu, se podría afirmar que todo rito tiende a consagrar o legitimar, es decir, "a desestimar en tanto que arbitrario y a reconocer en tanto que legítimo o natural, un límite arbitrario" (Bourdieu, 1986: 208). Es este hecho el que fundamentó la eficacia simbólica de los ritos, es decir "el poder de actuar sobre lo real, actuando sobre la representación de lo real" (1986: 2008). Declarar un bien patrimonio cultural, investirlo con esa denominación, ejerce una eficacia simbólica real en tanto que transforma realmente al bien consagrado. Es decir, transforma la imagen que de él tienen los demás agentes e incluso los comportamientos que adoptan con respecto a ella.

\section{A modo de cierre}

Díaz Cruz (2008) escribió: "Hay conceptos que pesan como lápidas (...) son conceptos con criterios fuertemente demarcacionistas (...) Otros, en cambio, no tienen esa pesadez, están permanentemente sujetos a debates, a réplicas y contrarréplicas, a desafíos" (2008: 37). E concepto de patrimonio cultural nació en la modernidad como "una especie de religión laica que sirve para sacralizar discursos en torno a las identidades sociales (Prats, 2006: 72). Su peso no daba lugar a dudas. Sus delimitaciones eran claras. Incluso en la actualidad el argumento de autoridad es avalado y legitimado por la ciencia, por lo que en el campo del patrimonio, la autoridad científica, en términos de dicho autor, es la que

en la mayoría de los casos, activa determinados referentes para que tengan efecto en lo social mediante un proceso de selección, ordenación e interpretación que conduce necesariamente a la formación de discursos patrimoniales, tradicionalmente destinados a sostener y sacralizar versiones ideológicas de la identidad que promueven adhesiones emotivas mediante la eficacia simbólica de los referentes patrimoniales (lbídem). 
La autoridad jurídica, por su parte, acrecienta la significación y legitimación al introducir al derecho en el campo de lo patrimonial elevándolo a una dimensión internacional. Sin embargo, y por los debates que se producen al interior de las ciencias sociales y desde perspectivas interdisciplinares, el concepto de patrimonio cultural inmaterial pierde el peso de una lápida pero no su carácter definitorio. De hecho, pensarlo en los términos de un campo y establecer la metáfora del juego para comprender cómo se establecen las relaciones en su interior, permite comprender como se redefinen los elementos que están en juego y en función de qué tipo de intereses.

En suma, pensar el patrimonio cultural inmaterial y la materialización de su definición en normativas legales y/o jurídicas a través de la noción de campo de Bourdieu permitió, en primer lugar, visibilizar al mismo en tanto producción socio cultural con el poder de inscribir significados y excluir otros. En segundo lugar, entender que la configuración de dichos significados no es aleatoria sino que responde y es producto de los intereses de quienes tienen el poder y conocen las reglas y aceptan jugar el juego. En tercer y último lugar, señalar su dimensión performativa y ritual posibilitó ir más allá de la descripción para comprender que existen lógicas que subyacen y que, al fin de cuentas, configuran el entramado de sentidos y relaciones que le dan forma y contenido al patrimonio cultural.

\section{Bibliografía}

Agudo Torrico, J. (2003). Patrimonio y derechos colectivos. Revista Antropología y patrimonio: investigación, documentación e intervención, 2, pp.12-29.

Blake, J. (2014). Convention for the Safeguarding of Intangible Cultural Heritage (2003). En Encyclopedia of Global Archaeology (pp. 1706-1711). Nueva York: Editorial Routledge.

Bonfil Batalla, G. (1989). Identidad nacional y patrimonio cultural: los conflictos ocultos y las convergencias. En R. Ceballos. (Comp). Antropología y políticas culturales. Patrimonio e identidad. Buenos Aires, Argentina: Departamento Nacional de Antropología y Folklore.

Bouchenaki, M. (2004). Editorial. Museum Internacional. Intangible Heritage, 32(221-222), pp. 7-12.

Bourdieu, P. (1993). Los ritos como actos de institución. En Pitt-Rivers, J. y Peristiany, J. (Eds.). Honor y gracia (pp. 111-123). Madrid, España: Alianza Universidad. 
Bourdieu, P. (2000). Elementos para una sociología del campo jurídico. En La fuerza del derecho. Bogotá, Colombia: Ediciones Uniandes y Siglo del Hombre Editores.

Bourdieu, P. (2001). La fuerza del derecho. Elementos para una sociología del campo jurídico. En Poder, derecho y clases sociales (pp. 101-129). España: Editorial Desclée.

Briones, C. (1998). (Meta)cultura del estado nación y estado de la (meta)cultura: repensando las identidades indígenas y antropológicas en tiempos de post- estatalizados. Universidad de Brasilia. Recuperado de http://www.dan.unb.br/images/doc/Serie244empdf.pdf

Canale, A. y Morel, H. (2005). Patrimonio intangible y políticas culturales: el caso de las murgas del carnaval de Buenos Aires. Cuadernos 21, pp. 111-132.

Da Matta, R. (1980). Carnavais, Malandros a Herois. Rfo de Janeiro: Ed. Zahar.

Dawson, M. (2004). Patrimonio Material e Inmaterial: de la Diferencia a la Convergencia. Museum Internacional. Intangible Heritage, 32(221-222), pp. 13-21.

Endere, M. y Mariano, M. (2013). Los conocimientos tradicionales y los desafíos de su protección legal en Argentina. Revista Quinto Sol, 17(2), pp.1-20.

Fortich Navarro, M. y Moreno Durán, A. (2012). Elementos de la teoría de los campos de Pierre Bourdieu para una aproximación al derecho en América Latina: consideraciones previas. Verba luris, 27, pp. 47-62.

Gavazzo, N. (2005). El patrimonio cultural boliviano en Buenos Aires: usos de la cultura e integración. En Martín, A. (Comp.). Folklore en las grandes ciudades. Arte popular, identidad y cultura (pp. 37-76). Buenos Aires, Argentina: Libros del Zorzal.

Geertz, C. (1974). The Interpretation of cultures. New York: Basic Books.

Geertz, C. (1994). Conocimiento local: hecho y ley en la perspectiva comparada. En Conocimiento local. Ensayos sobre la interpretación de las culturas (pp. 195-287). Barcelona, España: Paidós.

Gravano, A. (2008). La cultura como concepto central de la Antropología. En Chiringuini, M. (Comp.). Apertura a la antropología: alteridad, cultura, naturaleza humana (pp. 93-121). Buenos Aires, Argentina: Proyecto Editorial.

Higginbottom, G. (2014). Intangible cultural Heritage. Encyclopedia of Global Archaeology (pp. 3918-3921). Nueva York: Springer.

Kaufman, E. (1991). El ritual jurídico en el juicio a los ex comandantes. La desnaturalización de lo cotidiano. En Guber, R. (ed.). El salvaje metropolitano. Buenos Aires, Argentina: Legasa.

Kirchenblatt Gimblett, B. (2004). El patrimonio inmaterial como producción metacultural. Museum Intencional. Intangible Heritage, 32(221-222), pp. 52-67. 
Kurin, R. (2004). La salvaguarda del patrimonio cultural inmaterial en la Convención de la UNESCO de 2003: una valoración crítica. Museum Internacional. Intangible Heritage, 32(221-222), pp. 68-81.

Krotz, E. (2002). Sociedad, conflictos, cultura y derechos desde una perspectiva antropológica. En Antropología jurídica: perspectivas socioculturales en el estudio del derecho (pp.1346). Universidad Autónoma metropolitana-Iztapalapa, México: Editorial Anthropos.

Lacarrieu, M. (2010). ¿Es necesario patrimonializar las expresiones culturales "inmateriales? Desafíos teóricos y metodológicos en torno del patrimonio cultural "intangible". Actas $1^{\circ}$ Congreso Iberoamericano sobre patrimonio cultural. Experiencias metodológicas en el conocimiento del patrimonio, Universidad de Costa Rica.

Mariano, M. (2011). Patrimonio intangible e identidad: representaciones bolivianas en Olavarría, provincia de Buenos Aires, Argentina. Intersecciones en Antropología, 12, pp. 83-94.

Mariano, M. y Endere, M. (2013). Reflexiones acerca de la protección del patrimonio intangible a nivel internacional, regional y su proyección en Argentina. Dimensión Antropológica. 20(58), pp.33-60.

Mariotti, L. (2011). Patrimonio culturale immateriale: Un prodotto metaculturale. Ricerca Folklorica, 64, pp. 19-25.

Martin, A. (2001). El carnaval de Buenos Aires como patrimonio intangible. Un análisis desde la perspectiva del Folclore urbano. En Memorias, Identidades e Imaginarios Sociales (pp. 187-192). Buenos Aires: Secretaría de Cultura, gobierno de la ciudad de Buenos Aires.

Morel, H. (2007). Murgas y patrimonios en el carnaval de Bs. As. En Crespo, C.; Losada, F. y Martin, A. (Eds.). Patrimonio, políticas culturales y participación ciudadana (pp. 129144). Bs. As., Argentina: Antropofagia.

Moreno, A. (2003). Introducción elemental a Pierre Bourdieu, Bogotá, Colombia: Alvaro Moreno.

Organización Mundial de la Propiedad Intelectual (2008). Proyecto de Análisis de carencias en materia de Protección de los Conocimientos Tradicionales. Comité Intergubernamental sobre Propiedad Intelectual y Recursos Genéticos, Conocimientos Tradicionales y Folclore Recuperado de https://bit.ly/2KtpWQC

Prats, L. (2006). La mercantilización del patrimonio: entre la economía turística y las representaciones identitarias. Boletín de Instituto Andaluz del Patrimonio Hitórico, 58, pp. $72-80$.

Rotman, M. (2001). Legitimación y preservación patrimonial: la problemática de las manifestaciones culturales "no consagradas". Temas de Patrimonio, 5, pp. 151-168. 
Rotman, M. (2004). La creación urbana de tradiciones locales. En Rotman, M. (Ed.). Antropología de la cultura y el patrimonio. Diversidad y desigualdad en los procesos contemporáneos (pp. 135-151). Buenos Aires, Argentina: Ferreyra.

Rotman, M. (2010). El patrimonio de pueblos mapuches de Neuquén desde las perspectivas de sus habitantes, de las instituciones estatales y del mercado. En Hernández López, J. J.; Rotman, M. y González de Castells, A. (Eds.). Patrimonio y cultura en América Latina (pp. 19-34). Guadalajara: Universidad de Guadalajara.

Rotman, M. (2015). Procesos patrimoniales: redefiniciones, dinámica y tensiones en la contemporaneidad. Quehaceres. Revista del Departamento de Ciencias Antropológicas, 2. Facultad de Filosofía y Letras UBA, pp. 11-26.

Ruiz Muller, M. (2001). Protección de conocimientos, innovaciones y prácticas indígenas en el Perú. Debate Agrario: Análisis y alternativas, 33, Centro Peruano de Estudios Sociales, Lima, pp. 73-89. Recuperado de www.cepes.org.pe/debate/debate33/06-articuloda33.pdf

Sánchez-Carretero, C. (2005). Sobre el patrimonio inmaterial de la humanidad y la lucha por visibilizar "lo africano" en la República Dominicana. En Sierra Rodríguez, X. y Pereiro Pérez, X. (Coords.). Patrimonio Cultural: politizaciones y mercantilizaciones (pp. 147163). Sevilla, España: FFAAEE.

Tobón Franco, N. (2006). Un enfoque diferente para la protección de los conocimientos tradicionales de los pueblos indígenas. Revista Estudios Socio-jurídicos, 9(1), pp. 96129.

UNESCO (2003). Convención para la salvaguardia del patrimonio cultural inmaterial. Recuperado de https://bit.ly/2Hv2JQu

Van Zanten, W. (2004). La elaboración de una nueva terminología para el patrimonio cultural inmaterial. Museum International. Intangible Heritage, 32(221-222), pp. 36-43. 